\title{
Intervention study into the effect of feeding around exercise on fat oxidation in males
}

\author{
L. Turner, J. Over, S. Ford and A. Collins \\ Department of Nutritional Sciences, Faculty of Health and Medical Sciences, University of Surrey, Guildford, Surrey, \\ GU2 $7 T E$
}

Although much advice is given with regards to the timing of feeding around exercise for the purpose of improving performance ${ }^{(1)}$, few studies have compared the effect of carbohydrate (CHO) consumption before or after exercise on changes in metabolic fitness. Studies have suggested that males training with low carbohydrate can experience a higher contraction-induced response to exercise ${ }^{(2)}$. Acute findings have suggested that males may be able to improve their fat oxidation (FOx) rate by exercising in the post-absorptive state ${ }^{(3)}$ however, the chronic effect of this has yet to be investigated. Therefore the aim of this study was to determine the effect of repeatedly exercising or recovering in the post absorptive state on resting, exercise and post-exercise FOx levels. Ten healthy untrained male students aged 18-24yrs were recruited from the University of Surrey and randomly assigned and blinded to either training with CHO ingestion before (CHOPre; $n=5)$ or after (CHOPost; $n=5)$ each of six structured high intensity interval training sessions scheduled over two weeks. At baseline and three to four days after the last training session measurements of FOx were assessed in the fasted-rest, post-prandial exercise and the three hour post-exercise period by indirect calorimetry (breath-by-breath during exercise and ventilated hood during rest and recovery). Wilcoxon matched pairs tests, repeated measures two-way ANOVA tests and multiple t-tests were used to identify any significant differences from baseline to post-intervention in each of the intervention arms. This study protocol received a favourable ethical opinion from the University of Surrey (Ethics number EC/2011/41).

No significant differences in fasted basal FOx were seen as a result of training in either group $(P>0 \cdot 05$; data not shown). Similarly, FOx during moderate intensity exercise did not change significantly or show a trend following training in either group $(P>0.05$; data not shown). However, during the three hour post-exercise period, FOx significantly decreased following training in the CHOPre group (Fig. $1 ; P=0.02$ ), with no significant change in the CHOPost group (Fig.2; $P=0.56$ ).

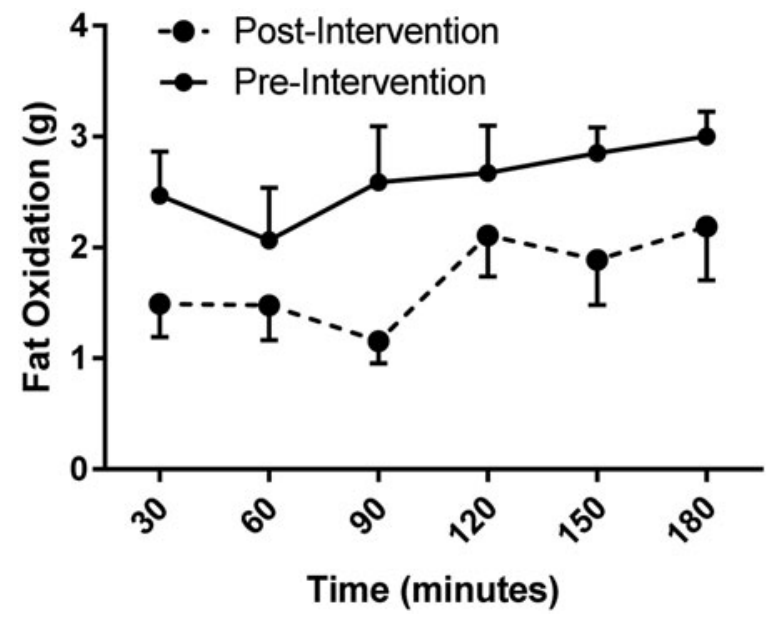

Fig. 1. 3 hour Post-exercise FOx in CHOPre Group.

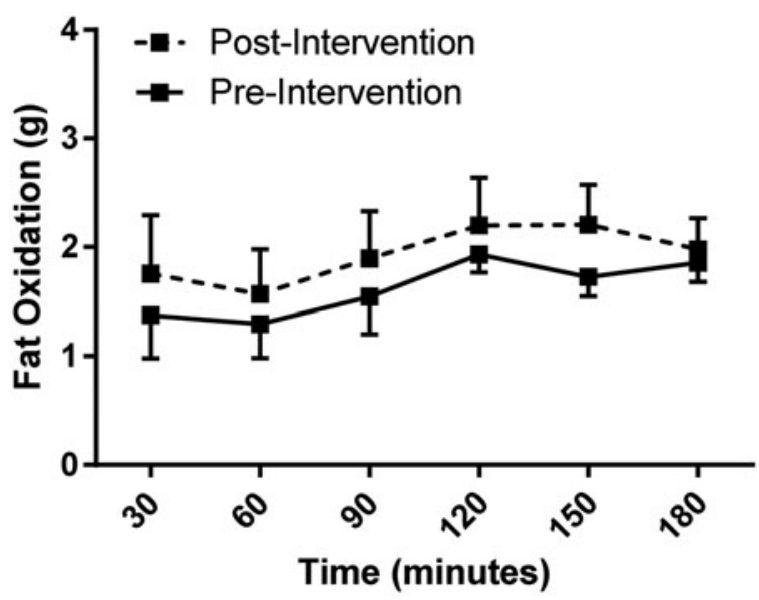

Fig. 2. 3 hour post-exercise FOx in CHOPost Group.

Overall the results allude to the fact that exercising in the fasted state and consuming $\mathrm{CHO}$ in the post-exercise period has little effect on FOx levels. In contrast, ingesting $\mathrm{CHO}$ prior to exercise may blunt or even counteract the effects of high intensity interval training to such an extent that FOx decreases as a result of training.

This study supports the notion that, for untrained males, consuming CHO after exercise is likely to be less detrimental to FOx levels than consumption prior to exercise. When advising exercising individuals who are aiming to improve metabolic health, these findings could have a potential public health implication.

1. Burke LM, Castell LM, Stear SJ et al. (2010) BJSM 44(5), 389-391.

2. Hawley JA, Burke LM, Phillips SM et al. (2011) J Appl Physiol. 110(3), 834-845.

3. Honnor M, Herdsman M, Collins AL. (2012) Proc Nutr Soc. 71(OCE3), E326. 